\title{
Polymer-Layered Silicate Nanocomposites: Synthesis, Properties and Applications
}

\author{
Emmanuel P. Giannelis \\ Department of Materials Science and Engineering, Cornell University, Ithaca, NY 14853, USA
}

Polymer nanocomposites, especially polymerlayered silicate (PLS) nanocomposites, represent a radical alternative to conventionally (macroscopically) filled polymers. Because of their nanometer-size dispersion, the nanocomposites exhibit markedly improved properties when compared with the pure polymers or conventional composites. These include increased modulus and strength, decreased gas permeability, increased solvent and heat resistance and decreased flammability. In addition to their potential applications, PLS nanocomposites are also unique model systems to study the structure and dynamics of polymers in confined environments. Using both delaminated and intercalated hybrids, the statics and dynamics of polymers confined over distances ranging from the radius of gyration of the polymer to the statistical segment length of the chains can be studied. (C) 1998 John Wiley \& Sons, Ltd.

Keywords: polymer; nanocomposites; silicates

Received 10 June 1997; accepted 2 October 1997

\section{INTRODUCTION}

Polymer nanocomposites, ${ }^{1}$ especially polymerlayered silicate nanocomposites, ${ }^{2}$ represent a radical alternative to conventionally (macroscopically) filled polymers. Because of their nanometer-size dispersion, the nanocomposites exhibit markedly improved properties when compared with the pure polymers or conventional composites. These include increased modulus and strength, decreased gas permeability, increased solvent and heat resistance and decreased flammability. For exam-

* Correspondence to: E. P. Giannelis, Department of Materials Science and Engineering, Cornell University, Ithaca, NY 14853, USA.

Contract/grant sponsor: Materials Science Center, Cornell University; Contract/grant number: NSF-DMR-MRSEC. ple, a doubling of the tensile modulus and strength is achieved for nylon-layered silicate nanocomposites with as little as 2 vol\% of inorganic content. ${ }^{3-5}$ In addition, the heat distortion temperature of the nanocomposites increases by up to $100{ }^{\circ} \mathrm{C}$, extending their use to higher-temperature environments, such as automotive under-the-hood parts. ${ }^{3}$ Furthermore, the heat release rate in the nanocomposites is reduced by up to $63 \%$ at heat fluxes of $50 \mathrm{~kW} \mathrm{~m}^{-2}$ without an increase in the $\mathrm{CO}$ and soot produced during combustion (J. W. Gilman and T. Kashiwagi, personal communication).

\section{SYNTHESIS OF NANOCOMPOSITES}

Melt intercalation of high polymers is a powerful new approach to the synthesis of polymer-layered silicate, (PLS) nanocomposites. ${ }^{6}$ This method is quite general and is broadly applicable to a range of commodity polymers, from essentially non-polar polystyrene to weakly polar poly(ethylene terephthalate) to strongly polar nylon. PLS nanocomposites can thus be processed using current technologies, and easily scaled to manufacturing quantities.

In general, two types of hybrid structures are possible: intercalated, in which a single, extended polymer chain is intercalated between the silicate layers, resulting in a well-ordered multilayer with alternating polymer/inorganic layers; and disordered or delaminated, in which the silicate layers (1) $\mathrm{nm}$ thick) are exfoliated and dispersed in a continuous polymer matrix (Figs 1 and 2).

\section{STRUCTURE AND PROPERTIES OF LAYERED SILICATES}

The silicates used belong to the general family of so-called 2:1 layered silicates. Their crystal structure consists of layers made up of two silica tetrahedra fused to an edge-shared octahedral sheet 


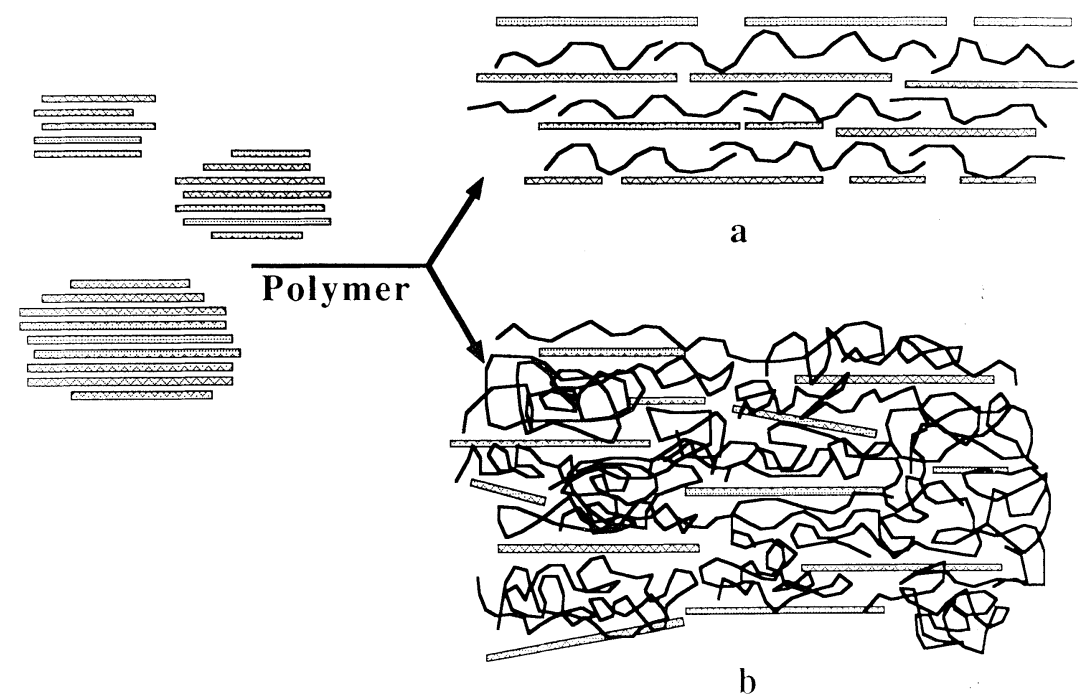

Figure 1 Schematic of composite structures obtained using layered silicates. The rectangular bars represent the silicate layers. (a) Single polymer layers intercalated in the silicate galleries (intercalated hybrids); (b) composites obtained by delamination of the silicate particles and dispersion in a continuous polymer matrix (delaminated hybrids).

of either alumina or magnesia. Stacking of the layers leads to a regular van der Waals gap between the layers called the interlayer or gallery. Isomorphic substitution within the layers generates negative charges that are normally counterbalanced by cations residing in the interlayer.

Pristine layered silicates usually contain hydrated $\mathrm{Na}^{+}$or $\mathrm{K}^{+}$ions. Ion-exchange reactions with cationic surfactants, including primary, tertiary and quaternary ammonium ions, render the normally hydrophilic silicate surface organophilic, which makes intercalation of many engineering polymers possible. The role of the alkylammonium cations in the organosilicates is to lower the surface energy of the inorganic component and improve the wetting characteristics with the polymer. Additionally, the alkylammonium cations can provide functional groups that can react with the polymer or initiate a polymerization of monomers to improve the strength of the interface between the inorganic component and the polymer.

\section{PROPERTIES OF NANOCOMPOSITES}

Polymer-layered silicate nanocomposites exhibit many advantages, including the following. ${ }^{2}$ (a) they are lighter in weight than conventionally filled polymers because high degrees of stiffness and strength are realized with far less high-density inorganic material: (b) they exhibit outstanding diffusional barrier properties without requiring a multipolymer-layered design, allowing for recycling; and (c) their mechanical properties are potentially superior to unidirectional fiber-reinforced polymers, because reinforcement from the inorganic layers will occur in two dimensions rather than in one.

Recent work at the National Institute of Standards \& Technology (NIST) has also shown that the nanocomposites have excellent flammability properties. The heat release rate measured by cone calorimetry is reduced by $63 \%$ in a nylon- 6 silicate nanocomposite containing $2 \mathrm{vol} \%$ of silicate. In addition, in contrast to many commercial fire retardants, the rate of soot and $\mathrm{CO}$ generation does not increase during combustion. The flame-retardant behavior of the nanocomposites has been attributed to the formation of a tough char layer which may act as a mass-transport barrier, slowing the escape of volatile by-products during combustion (J. W. Gilman and T. Kashiwagi, personal communication).

Similar improved char yields and flame resistance have also been observed by us for polycaprolactone, $^{2}$ polyether imide ${ }^{7}$ and vinyl esterlayered silicate nanocomposites (J. D. Lee and E. P. 


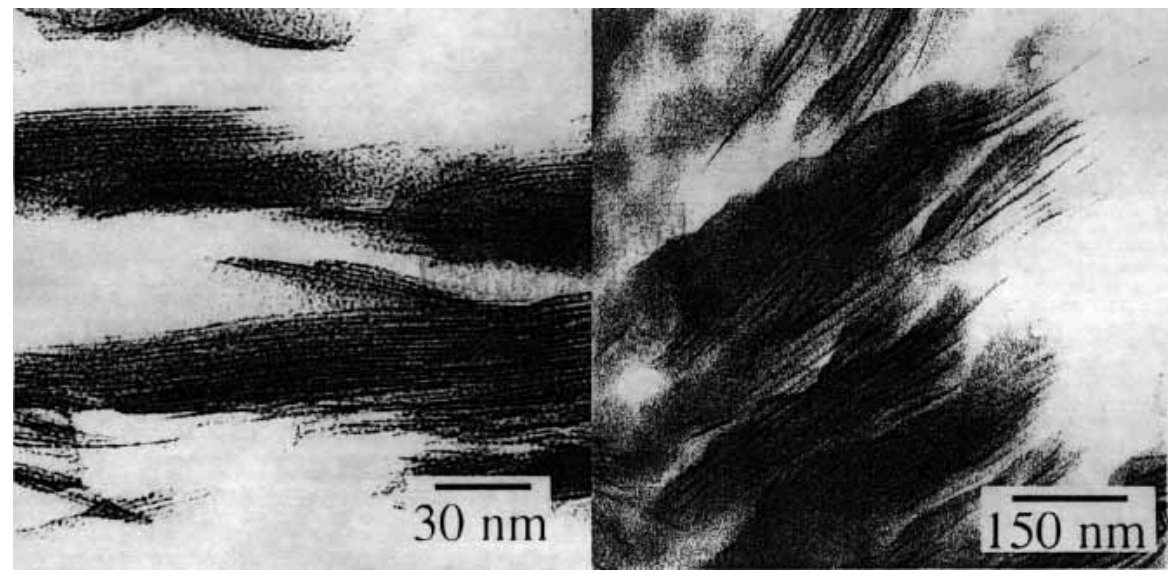

Figure 2 Transmission electron micrographs of a polystyrene intercalated nanocomposite (left) and an epoxy delaminated nanocomposite (right).

Gianellis, unpublished results). For example, polyether imide nanocomposites retain about 90 and $45 \%$ of their weight after 20 and $120 \mathrm{~min}$, respectively, at $450{ }^{\circ} \mathrm{C}$. The respective values for the pure polymer are 45 and $15 \%$. In the $500^{\circ} \mathrm{C}$ isothermal the polymer is completely lost after $40 \mathrm{~min}$, while the char yield of the nanocomposite is $\mathrm{ca} 55 \%{ }^{8}$. In addition, when samples are exposed to an open flame, burning of the pure polymer persists after the flame is removed, until it is externally extinguished. In contrast, the nanocomposites stop burning after the flame is removed and become highly charred but maintain their initial dimensions. It is important to note that the nanodispersion of the silicate is essential to this flame-retardant behavior. Polymers macroscopically filled with the same silicate as in the nanocomposites and at equivalent loadings show at best the same, and in some cases an increase (rather than a decrease), in polymer flammability. ${ }^{8}$

In contrast to other flame-retardant additives which tend to deteriorate in mechanical properties, the nanocomposites show the opposite trend. Table 1 summarizes some of the properties of nylon- 6 nanocomposite containing only $2 \mathrm{vol} \%$ silicate (similar to the sample used in the cone calorimeter experiments). ${ }^{3}$ Typically, the same improvements in properties of conventionally filled nylon require additions of $\mathrm{ca} 30 \mathrm{vol} \%$ mineral.

In addition to the mechanical properties, the barrier properties of the nanocomposites are dramatically improved compared with the pure or macroscopically filled polymers, even at much higher filler content (Fig. 3). The dramatic reduction in permeability has been attributed to the presence of well-dispersed silicate layers with a large aspect ratio which causes solutes to follow a tortuous path, thereby increasing the effective path length for diffusion. ${ }^{2}$

\section{MISCIBILITY/THERMODYNAMICS OF NANOCOMPOSITE FORMATION}

Similarly to polymer blends, any mixture of polymer and layered silicate does not necessarily lead to a nanocomposite. In most the incompatibility of the hydrophobic polymer and the hydrophilic silicate leads to phase separation similar to that of macroscopically filled systems. In contrast, by using surface-modified silicates, as noted earlier, one can fine-tune their surface energy and render them miscible (or compatible) with different polymers. The approach is based on a chemical (rather than a mechanical) driving force which leads to nanoscopic dispersion.

What is then the driving force for polymer intercalation which leads to nanodispersion? To answer this question we have developed recently a mean-field model. ${ }^{4}$ Nanocomposites are formed if the free energy change, $\Delta G$, of the process is negative. The change in free energy is composed of an enthalpic term, $\Delta H$, due to the intermolecular interactions and an entropic term, $\Delta S$, associated with the configurational changes of the constituents, and $\Delta G=\Delta H-T \Delta S$, where $T$ is the temperature. The entropy change of the organically modified inorganic component is calculated using a modified Flory-Huggins lattice model in which 
Table 1 Properties of nylon-6 and silicate-nylon nanocomposites ${ }^{\mathrm{a}}$

\begin{tabular}{lcc}
\hline Property & Nanocomposite & Nylon-6 \\
\hline Tensile modulus $(\mathrm{GPa})$ & 2.1 & 1.1 \\
Tensile strength $(\mathrm{MPa})$ & 107 & 69 \\
Heat distortion temp. $\left({ }^{\circ} \mathrm{C}\right)$ & 145 & 2.3 \\
Impact strength $\left(\mathrm{kJ} \mathrm{m}^{-2}\right)$ & 2.8 & 0.87 \\
Water adsorption $(\%)$ & 0.51 & $13 \times 10^{-5}$ \\
Coefficient of thermal expansion & $6.3 \times 10^{-5}$ & \\
\hline
\end{tabular}

\section{${ }^{a}$ Ref. 3.}

the occupation of the lattice is weighted to simulate the preferred orientations of the organic modifier (tethered chains) in the presence of two impenetrable surfaces (silicate layers). The confinement of the polymer chains is similarly approximated using a self-consistent field treatment of a random-flight polymer between two surfaces. For the enthalpic term a modified mean-field, site-fraction approach where the number of contacts per lattice site is replaced by an interaction area per lattice site is being used. This approach makes it possible to express the interaction parameter as energy per area and may be approximated by interfacial or surface energies.

Using this model we find that the entropy loss associated with the polymer confinement is ap- proximately compensated by an entropy gain associated with the increased conformational freedom of the surfactant molecules as the gallery distance increases due to the polymer intercalation. Therefore, enthalpy determines whether or not polymer intercalation will take place. As the polymer-silicate interactions become stronger, first intercalated and then delaminated nanocomposites are predicted, in agreement with the experiment. ${ }^{9}$

\section{STRUCTURE AND DYNAMICS OF POLYMER NANOCOMPOSITES}

In addition to their potential applications, PLS

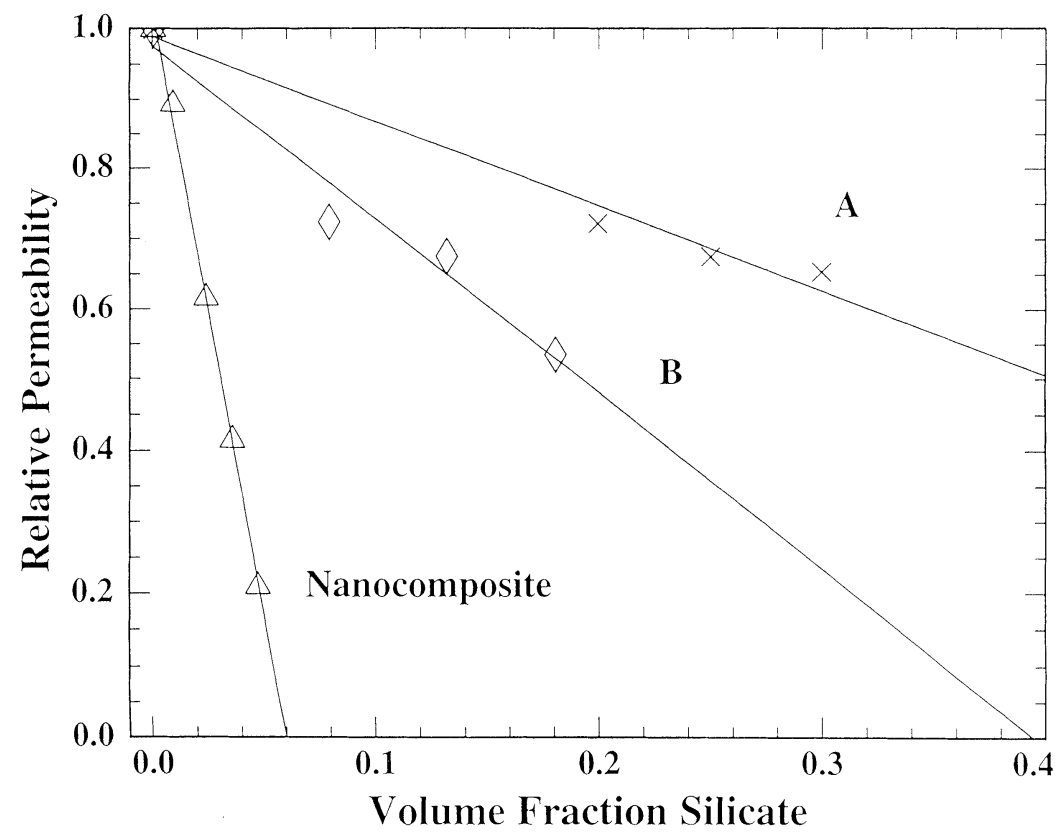

Figure 3 Relative permeability as a function of inorganic content of (A) polycaprolactone nanocomposites and (B) conventionally filled (i.e. without nanodispersion) polymers. ${ }^{2}$ 


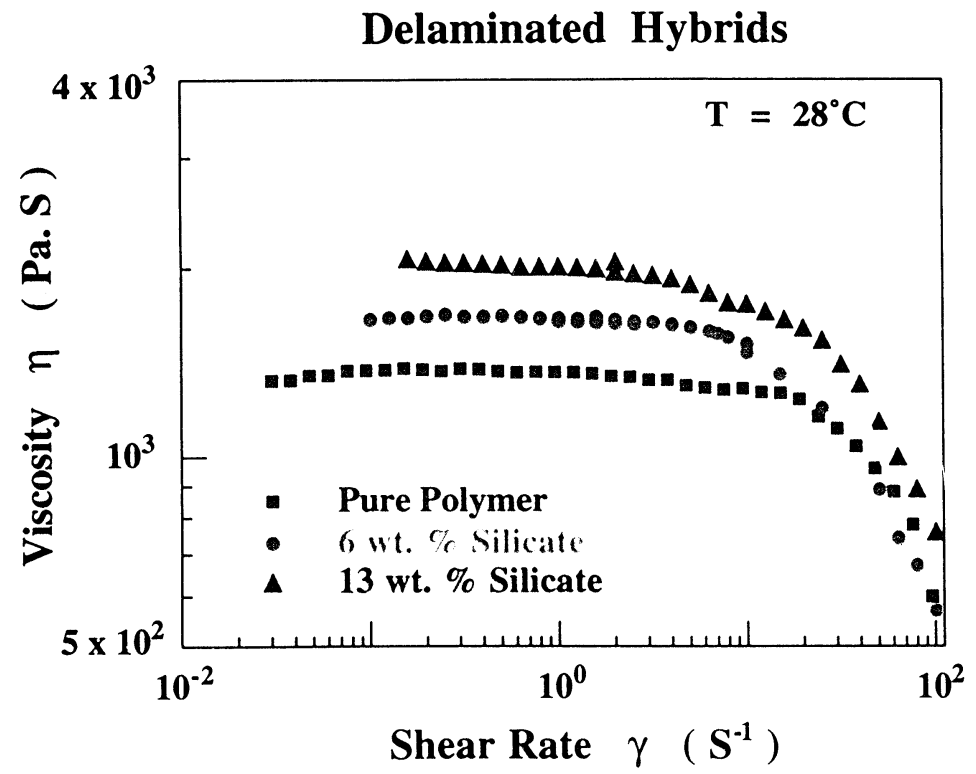

Figure 4 Steady-state shear viscosity as a function of shear rate for polydimethylsiloxane layered silicate nanocomposites containing different levels of silicate loadings. ${ }^{10}$

nanocomposites are unique model systems to study the statics and dynamics of polymers in confined environments. ${ }^{10}$ Generally, the dynamics and structure of polymers in confined environments are a fundamental aspect of many industrially important fields such as tribology, adhesion, lubrication, sorption and catalysis. Using both delaminated and intercalated hybrids, the statics and dynamics of polymers confined over distances ranging from the radius of gyration of the polymer to the statistical segment length of the chains can be studied.

In general, the behavior of polymer liquids under confinement is very different from that in the bulk, especially when the polymer thickness approaches the size of the polymer coil. Traditional notions such as reptation dynamics governing the relaxation of a long polymer chain are improbable in highly confined intercalated systems (confinement distances comparable with the statistical segment length of the polymer), as it is difficult to imagine an entanglement in two dimensions.

Despite the topological constraints imposed by the host lattice, mass transport of the polymer into the silicate layers appears to be unhindered and exhibits mobility which is faster than the selfdiffusion of the pure polymer. ${ }^{11}$ The observation that polymer chains can undergo center-of-mass transport in essentially two dimensions is rather surprising because the unperturbed radius of gyration of the polymer is roughly an order of magnitude greater than the interlayer distance between the silicate layers. The ability of the chains to undergo center-of-mass transport during melt intercalation is evidence that the interactions within the interlayer does not completely restrict segmental motions, otherwise large-scale chain motion would not be possible.

In addition, the dynamics of the polymer in the hybrids are dramatically different from those in the bulk. On a local scale, intercalated polymer chains exhibit higher flexibility along their backbone, together with a marked suppression (or even absence) of the cooperative dynamics typically associated with the glass transition.

Finally, since most fabrication techniques involve flow, we briefly address the rheology of nanocomposites. In contrast to macroscopically filled polymers, in which filler addition leads to dramatic increases in viscosity, silicate nanocomposites exhibit shear thinning. ${ }^{11}$ For example, the steady-state shear viscosity of a series of polydimethylsiloxane nanocomposites shows an increase with respect to that of the pure polymer at low shear rates but still obeys Newtonian-type behavior, even at reasonably high silicate loadings 
(Fig. 4). ${ }^{11}$ The same effect is observed in smallamplitude oscillatory shear measurements, wherein the storage and loss moduli for the nanocomposites display similar frequency dependence to the pure polymer, with a monotonic increase in the magnitude of the moduli with increasing silicate content. Although at present the exact mechanism that causes shear thinning is not known, we suspect that it is due to the orientation of the silicate layers under shear and polymer coils [12]. Nevertheless, because of this shear thinning the nanocomposites can be melt-processed, and this simplifies their manufacturing.

Acknowledgments This work was supported by the Materials Science Center at Cornell (NSF-DMR-MRSEC) and by generous gifts from Dupont, Eastman, Exxon, Monsanto, Nanocor, Southern Clay Products and Xerox.

\section{REFERENCES}

1. B. M. Novak, Adv. Mater. 5, 422 (1993).
2. E. P. Giannelis, Adv. Mater. 8, 29 (1996).

3. A. Usuki, M. Kawasumi, Y. Kojima, A. Okada, T. Kurauchi and O. Kamigaito, J. Mater. Res. 8, 1174 (1994).

4. Y. Kojima, A. Usuki, M. Kawasumi, A. Okada, T. Kurauchi and O. Kamigaito, J. Polym. Sci. Part A: Polym. Chem. 31, 983 (1993).

5. K. Yano, A. Usuki, T. Karauchi et al. J. Polym. Sci.: Part A: Polym. Chem. 31, 2493 (1993).

6. R. A. Vaia, H. Ishii and E. P. Giannelis, Chem. Mater. 5, 1694 (1993).

7. J. D. Lee, T. Takehoshi and E. P. Giannelis, Fire resistant nanocomposites. In: Nanophase and Nanocomposite Materials II Komarneni, S., Parker, J. C. and Wollenberger, H. J. (eds), MRS Symp. Proc. 457, Pittsburgh, PA, 1997, pp. 513-518.

8. M. LeBras, Fire and Materials 20, 39 (1996).

9. R. A. Vaia and E. P. Giannelis, Macromolecules 30, 7990 (1997); Macromolecules 30, 8000 (1997).

10. R. Krishamoorti, R. A. Vaia and E. P. Giannelis, Chem. Mater. 8, 1728 (1996).

11. R. A. Vaia, K. D. Jandt, E. J. Kramer and E. P. Giannelis, Macromolecules 28, 8080 (1995).

12. E. Manias, G. Hadziioannou and G. ten Brinke, Langmuir 12, 4587 (1996). 